\title{
93. Deutscher Röntgenkongress vom 16. bis 19. Mai 2012 in Hamburg
}

Der diesjährige Deutsche Röntgenkongress rückt näher, deshalb darf ich Sie auf einige Höhepunkte nochmals besonders hinweisen.

\section{DRG trifft DGN}

Im Rahmen der fachübergreifenden Sitzungen unter der Überschrift „DRG trifft...“ ist die Veranstaltung zusammen mit der Deutschen Gesellschaft für Nuklearmedizin (DGN) besonders hervorzuheben. So wurde zwischen den Vorständen beider Fachgesellschaften vereinbart, die gegenseitige Zusammenarbeit zu intensivieren und z.B. gemeinsame Veranstaltungen beim Deutschen Röntgenkongress ebenso wie bei der Jahrestagung der DGN durchzuführen. Ansatzpunkte hierfür sind im medizinischen Bereich die Hybridbildgebung, im Ausbildungsbereich im jeweils anderen Fachgebiet zu erwerbende Fachkunden im Strahlenschutz.

Als Besonderheit dieser Schwerpunkt-Sitzung ist anzusehen, dass die Themen von je einem Redner aus radiologischer und nuklearmedizinischer Sicht beleuchtet werden. So informiert der 1 . Block unter dem Titel „Hybridbildgebung“ über den aktuellen Stand der MR-PET, den Vergleich von PET-CT und Ganzkörper-MRT und den Stellenwert der radiologischen bzw. nuklearmedizinischen Untersuchungsverfahren in der Mammadiagnostik. Im 2. Veranstaltungsabschnitt mit dem Motto „Neue Trends für die Praxis“ beleuchten die Vortragenden neue Tracerentwicklungen auf der Basis des Fluor-18, definieren den Stellenwert der bildgebenden Verfahren in der Herzdiagnostik im Jahr 2012 und gehen der Frage nach, welche Klassifikation in der onkologischen Response-Beurteilung aktuell verfügbar und empfehlenswert ist (s. Kasten).

\section{Röntgenvorlesung $\nabla$}

Einen seiner akademischen Höhepunkte findet jeder Deutsche Röntgenkongress in der jährlichen Röntgenvorlesung. Hierzu lädt der Kongresspräsident, einer guten Tradition folgend, einen herausragenden und international renommierten Wissenschaftler und radiologischen Lehrer ein. In diesem Jahr wird Richard L. Baron, Dean for Clinical Practice und Professor of Radiology an der Universität von Chicago/USA zum Thema „The Evolution of Liver Imaging: The Search for the Holy Grail" sprechen. Prof. Baron ist ein seit vielen Jahren anerkannter Experte auf dem Gebiet der Abdominal- und speziell der Leberradiologie. So war er einer der ersten, der in einer echten ,lesion-by-lesion“Analyse die Ergebnisse der Schnittbilddiagnostik mit den Pathologiebefunden an explantierten Organen korrelierte. So sind von Prof. Barons Ausführungen sicherlich spannende Hinweise zum Stellenwert der bildgebenden Diagnostik der Leber, ihren Möglichkeiten und Grenzen zu erwarten. Darüber hinaus impliziert der Titel jedoch auch am Beispiel der hepatischen Bildgebung eine Definition der Rolle des Radiologen bei Diagnostik und Therapie unserer Patienten.

Zugleich werden im Rahmen der akademischen Feier der Röntgenvorlesung die hochrangigen Preise der Deutschen Röntgengesellschaft verliehen (s. Kasten).

\section{Get together | Eröffnungs- veranstaltung $\checkmark$}

Erstmals werden beim 93. Deutschen Röntgenkongress das abendliche gesellschaftliche Zusammentreffen am Anreisetag und die offizielle akademische Eröffnungsfeier getrennt.

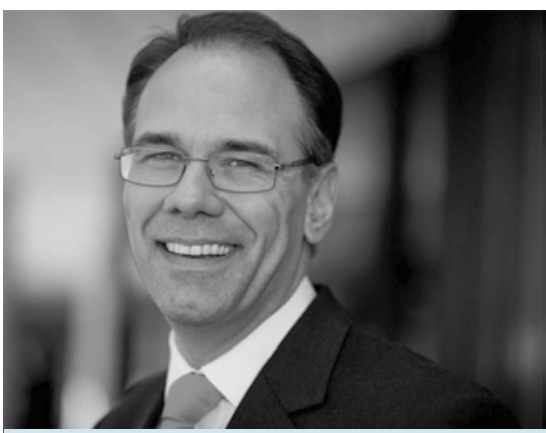

Prof. Dr. Hermann Helmberger

Für das „Get together“ am Mittwochabend wurde als Ort der „Schuppen 52“, ein aktuell sehr beliebter Veranstaltungsort im Hamburger Hafen, gewählt. Kommen Sie zusammen mit Ihren Kollegen und Mitarbeitern, lassen Sie sich anstecken vom Flair der alten Kaufmanns- und Hafenstadt Hamburg, treffen Sie Freunde, Kollegen, Referenten, Kongressteilnehmer vom Studenten bis zum Lehrstuhlinhaber in lockerer und entspannter Atmosphäre als Einstimmung auf die folgenden Kongresstage. Nähere Informationen und die Anmeldeformalitäten entnehmen Sie bitte der nachstehenden Ankündigung.

Die offizielle Kongresseröffnung mit einer Einstimmung auf das Angebot und die medizinischen Schwerpunkte findet dann am Donnerstagvormittag nach dem 1. Refresher-Block ohne Überschneidung mit anderen Veranstaltungen statt. Im Rahmen dieser Feier werden auch die diesjährigen Ehrenmitgliedschaften der DRG verliehen. Bereits heute darf ich Sie hierzu, auch im Namen des Vorstands, aber auch ganz persönlich sehr herzlich einladen.

\section{Weitere Informationen $\nabla$}

Aktuelle Informationen unmittelbar vor und zum Kongress erfahren Sie über den Newsletter der DRG, der jedem DRG-Mitglied elektronisch zugeht, über den elektronischen Newsletter der RöFo, zu dem Sie 
sich kostenlos registrieren können und über unsere Kongresszeitung „RÖKO HEUTE“, die Sie tagesaktuell in Hamburg von Mittwoch bis Freitag erhalten werden.

Ich freue mich, Sie in wenigen Tagen in Hamburg zum Deutschen Röntgenkongress 2012 begrüßen und treffen zu können und wünsche Ihnen eine gute und sichere Anreise.

Prof. Dr. Hermann Helmberger Präsident des 93. Deutschen Röntgen-

kongresses 